\title{
Esclerite posterior associada à oclusão da veia central da retina e edema macular cistoide: relato de caso
}

\author{
Posterior scleritis associated with central retinal vein occlusion and cystoid macular edema: case report
}

ROger WAdA KAMEI ${ }^{1}$

\section{RESUMO}

Apresentamos um caso de esclerite posterior associada à oclusão da veia central da retina e edema macular cistoide. Com esse caso, observamos como a doença pode ser agressiva e como a acuidade visual pode ser comprometida. No entanto, o tratamento correto pode melhorar as alterações oculares com melhora visual. Alertamos também para os cuidados que devem ser tomados em relação ao uso de altas doses de corticosteroides.

Descritores: Esclerite/quimioterapia; Oclusão da veia retiniana; Edema macular; Uveítes; Angiofluoresceinografia; Metilprednisolona/uso terapêutico; Relato de caso; Humanos; Idoso; Feminino

\begin{abstract}
A case of posterior scleritis associated with central retinal vein occlusion and cystoid macular edema is reported. With this case, we noticed how the disease can be aggressive and how the visual acuity can be compromised. However, the correct treatment can improve the ocular changes with visual improvement. We also alert to the care with the use of high doses of corticosteroids.
\end{abstract}

Keywords: Scleritis/drug therapy; Retinal vein occlusion; Macular edema; Uveitis; Fluorescein angiography; Methylprednisolone/therapeutic use; Case report; Humans; Aged; Female

\section{INTRODUÇÃO}

A esclerite posterior é uma forma rara de esclerite que, apesar da sua grande morbidade, apresenta uma grande dificuldade no seu diagnóstico. Definida como um processo inflamatório da esclera posterior até a ora serrata com sintomas que, frequentemente, simulam outras condições, sendo uma doença pouco diagnosticada ${ }^{(1-3)}$

Como em outras formas de esclerites, a dor é um sintoma importante e a visão pode ser gravemente afetada. Além do quadro clínico, a ecografia B mostra-se de grande utilidade para confirmar o diagnóstico, achados ecográficos são muito comuns e fundamentais para o acompanhamento desses $\operatorname{casos}^{(1,2)}$, principalmente quando não há sinais fundoscópicos.

Em geral acomete mais mulheres (60 a 70\%) que homens, entre 40 e 59 anos, sendo bilateral em 5\% dos casos e com recorrências frequentes. Podendo estar associada à esclerite anterior em 43,5\%, principalmente na forma difusa ${ }^{(4-6)}$.

A esclerite posterior pode ser idiopática ou estar associada a uma doença sistêmica em até $40 \%$ dos casos. Causas infecciosas podem estar presentes, como herpes zoster, herpes simples, tuberculose e sífilis. Porém, a artrite reumatóide é descrita como sendo a associação mais comum. Outras vasculites sistêmicas como granulomatose de Wegener também podem ser uma causa ${ }^{(7,8)}$.

O tratamento depende do diagnóstico correto e, em casos mais graves e não responsivos ao tratamento convencional com drogas anti-inflamatórias, pode ser necessário o uso de drogas imunossupressoras para o controle do quadro ocular ${ }^{(9,10)}$

Uma abordagem multidisciplinar desses casos, muitas vezes, é necessária. Sendo uma avaliação clínica geral e uma investigação laboratorial precoce de extrema importância. O encaminhamento para o reumatologista é aconselhável para cooperação no diagnóstico e tratamento.

O processo inflamatório escleral pode ser potencialmente devastador. O não reconhecimento e não tratamento dessa condição pode comprometer a visão de maneira rápida e irreversível.

\section{RELATO DE CASO}

A.M.B., sexo feminino, 71 anos. Iniciou quadro de baixa acuidade visual (AV), hiperemia e dor no olho direito (OD) há dois meses, desde então, com diagnóstico e tratamento de conjuntivite. Referia ser "pré-diabética" controlada com dieta, sem uso de medicação hipoglicemiante. Negava hipertensão arterial sistêmica e trauma.

A AV era de movimentos de mãos frente os olhos em OD e de 20/25 em OE. À biomicroscopia, o OD apresentava quadro de esclerite anterior difusa, com grande congestão dos vasos episclerais superficiais e profundos, ausência de reação de câmara anterior (RCA), pressão intraocular normal e pseudofacia sem complicações. O olho esquerdo (OE) mostrava-se sem alterações.

A fundoscopia do OD (Figura 1) mostrava um descolamento seroso da retina com descolamento periférico da coroide e ausência de roturas retinianas. O vítreo estava claro com raras células inflamatórias. Havia um edema do disco óptico importante com sinais compatíveis com oclusão da veia central da retina (OVCR) com hemorragias retinianas superficiais, dilatação e ingurgitamento venoso significativo, alguns exsudatos algodonosos e mácula sem edema. OE normal.
Submetido para publicação: 21 de março de 2012

Aceito para publicação: 9 de outubro de 2012

Trabalho realizado no Instituto Penido Burnier.

Médico, Setor de Retina e Vítreo, Uveítes e AIDS, Instituto Penido Burnier, Campinas (SP), Brasil.
Financiamento: Não houve financiamento para este trabalho.

Divulgação de potenciais conflitos de interesse: R.Kamei, Nenhum.

Endereço para correspondência: Roger Kamei. Rua Coelho Neto, 15 - Apto. 54 - Campinas (SP) 13023-020 - Brasil - E-mail: rwkamei@gmail.com 
O ultrassom mostrava um vítreo pouco acometido e sem trações vitreorretinianas anormais, descolamento de coroide periférico, descolamento seroso da retina e edema do disco óptico perceptível ao exame. A coroide apresentava um espessamento importante e difuso sem, no entanto, apresentar acúmulo de líquido no espaço subtenoniano. Não foi observado o característico "sinal do T invertido". O exame também afastou a presença de massas e demais alterações da parede ocular.

A angiofluoresceinografia (AGF) mostrava o grande comprometimento vascular retiniano e ausência de edema macular (Figura 2).

Neste momento, foram solicitados exames laboratoriais infecciosos e reumatológicos, assim como uma avaliação clínica para liberação para pulsoterapia com metilprednisolona endovenosa por três dias consecutivos. Os exames laboratoriais mostraram negatividade para toda a investigação sorológica e as condições clínicas da paciente permitiram a realização da pulsoterapia. O tratamento foi realizado em regime de internação hospitalar e monitorização clínica.

Com a medicação endovenosa, obteve-se resolução total do descolamento da coroide e parcial do descolamento seroso da retina. Sendo programada a manutenção do tratamento com prednisona oral $1 \mathrm{mg} / \mathrm{kg} / \mathrm{dia}$, porém os índices glicêmicos da paciente se elevaram para valores acima de $300 \mathrm{mg} / \mathrm{dL}$. Assim, permaneceu internada para controle da glicemia por 20 dias, tendo alta com uso de insulina diária três vezes ao dia e hipoglicemiantes orais, mantendo glicemia

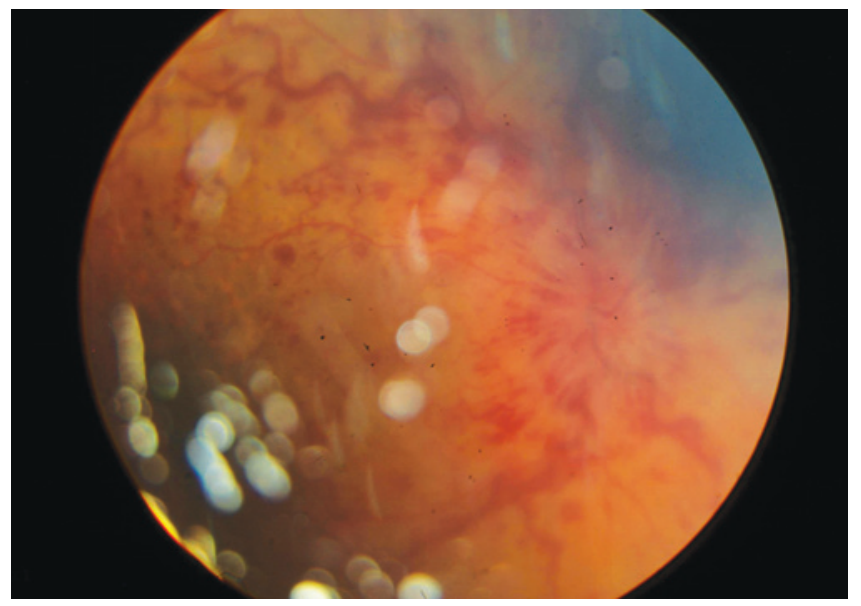

Figura 1. Retinografia colorida do OD inicial.

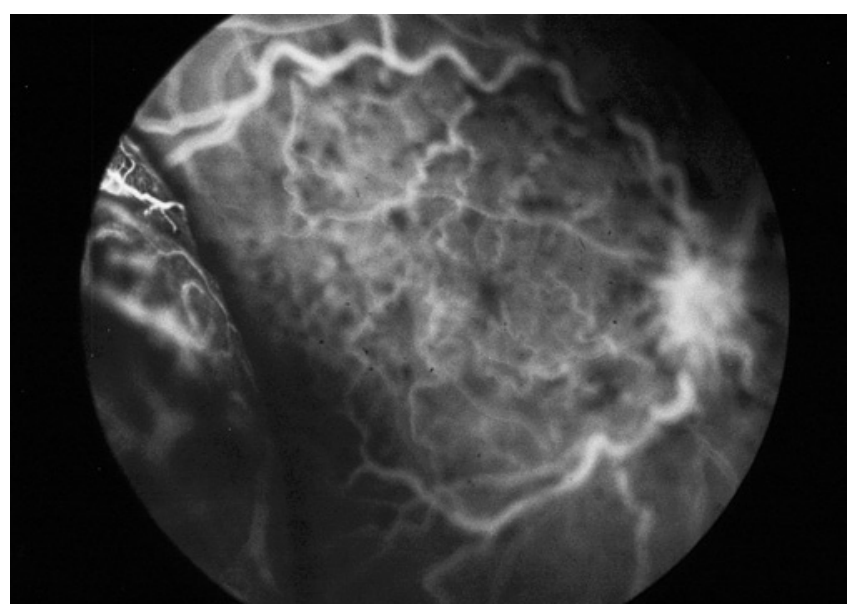

Figura 2. Angiofluoresceinografia do OD. de 200 mg/dL e 20 mg de predinisona por dia. Neste momento, OD estava com a retina totalmente colada, mantendo o edema do disco óptico e melhora parcial dos sinais da OVCR, porém com AV de 20/200.

Com a realização da tomografia de coerência óptica $(O C T)$ e nova AGF, notou-se um edema macular de $1.075 \mu \mathrm{m}$ na área central (Figura 4), também evidenciado pelo exame angiográfico.

Devido às condições clínicas da paciente, optou-se pelo tratamento do edema macular com aplicação de corticosteroide intraocular de acetato de triancinolona. Após a realização de duas injeções com intervalo de 40 dias, o edema macular apresentou completa resolução (245 $\mu \mathrm{m}$ ) e a AV melhorou para 20/25. Ao final, a retina estava totalmente colada, sem edema do disco óptico e sinais de OVCR (Figura 3).

\section{CONCLUSÃO}

Com esse relato, podemos perceber como a esclerite posterior pode ser agressiva e exigir um tratamento intenso a base de corticosteroide. No entanto, essa condição pode ser erroneamente diagnosticada e levar a sérias complicações decorrentes da inflamação ocular.

Em relação ao tratamento com corticosteroides, nota-se um grande cuidado principalmente em pacientes idosos e com comorbidades. Atenção especial deve ser dada à diabetes melito e hipertensão arterial sistêmica. Nesses casos, pode-se pensar em outras opções de tratamento, como a aplicação intra e peri-ocular e imunossupres-

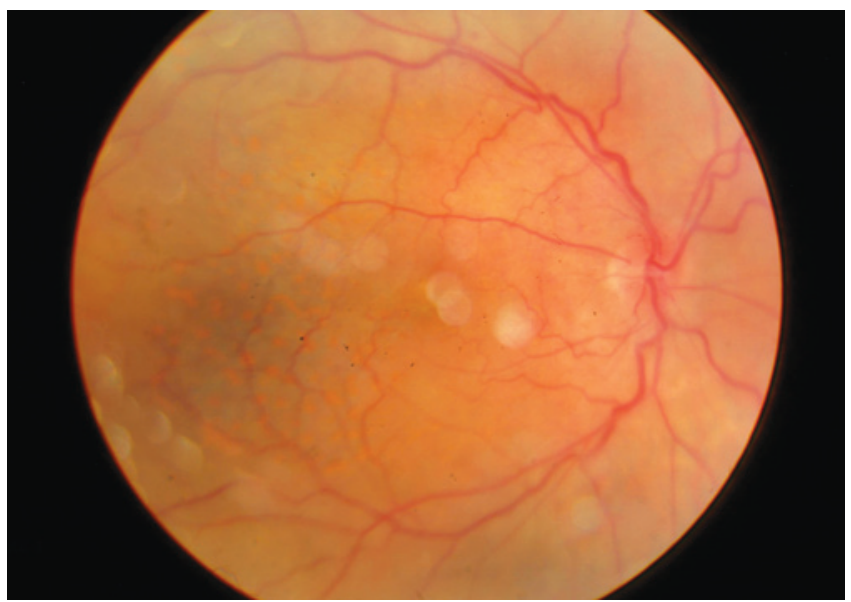

Figura 3. Retinografia colorida do OD após tratamento.

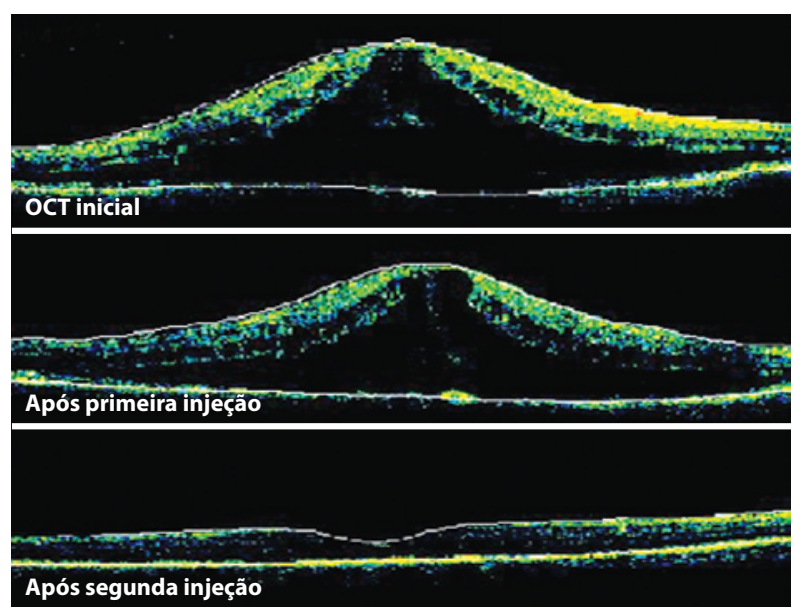

Figura 4. Evolução do edema macular pelo OCT durante o tratamento. 
sores sistêmicos. Dessa forma, uma abordagem multidisciplinar com um reumatologista e um clínico geral é de extrema importância para o manejo da medicação e acompanhamento desses casos.

Apesar do quadro agressivo que a esclerite posterior pode proporcionar, o correto tratamento com altas doses de corticosteroides pode apresentar grande melhora e até resolução das alterações oculares.

Percebe-se, assim, a importância do diagnóstico precoce e pronto tratamento da esclerite posterior. Sendo uma condição grave, onde medicações sistêmicas e uma abordagem multidisciplinar podem ser necessárias para uma melhor resposta do quadro inflamatório ocular.

\section{REFERÊNCIAS}

1. McCluskey PJ, Watson PG, Lightman S, Haybittle J, Restori M, Branley M. Posterior scleritis: clinical features, systemic associations, and outcome in a large series of patients. Ophthalmology. 1999;106(12):2380-6.

2. Benson WE. Posterior scleritis. Surv Ophthalmol. 1988;32(5):297-316.
3. Machado DO, Curi AL, Bessa TF, Campos WR, Oréfice F. Esclerite posterior: características clínicas, associação sistêmica, tratamento e evolução de 23 pacientes. Arq Bras Oftalmol. 2009;72(3):321-6.

4. Parra AG, Miyazaki FH, Ribeiro RM, Gehlen ML, Skare, T. Análise de 29 casos de esclerite. Experiência de um serviço de Reumato-Oftalmologia. Arq Bras Oftalmol. 2010;73(3):250-3.

5. Machado DO, Curi AL, Fernandes RS, Bessa TF, Campos WR, Oréfice F. Esclerite: características clínicas, associação sistêmica, tratamento e evolução de 100 pacientes. Arq Bras Oftalmol. 2009;72(2):231-5

6. La Maza MS, Molina N, Gonzalez-Gonzalez LA, Doctor PP, Tauber J, Foster CS. Clinical characteristics of a large cohort of patients with scleritis and episcleritis. Ophthalmology 2012;119(1):43-50. Comment in: Ophthalmology. 2012;119(8):1715-1715.e.1.

7. Raiji VR, Palestine AG, Parver DL. Scleritis and systemic disease association in a community-based referral practice. Am J Ophthalmol. 2009;148(6):946-50.

8. Akpek EK, Thorne JE, Qazi FA, Do DV, Jabs DA. Evaluation of patients with scleritis for systemic disease. Ophthalmology. 2004;111(3):501-6. Comment in: Ophthalmology. 2007;114(6):1232.

9. Jabs DA, Mudun A, Dunn JP, Marsh MJ. Episcleritis and scleritis: clinical features and treatment results. Am J Ophthalmol. 2000;130(4):469-76.

10. Doctor P, Sultan A, Syed S, Christen W, Bhat P, Foster CS. Infliximab for the treatment of refractory scleritis. Br J Ophthalmol. 2010;94(5):579-83. 